\title{
Lane Change Control Combined with Ramp Metering: A Strategy to Manage Delays at On-Ramp Merging Sections
}

\author{
Hari Hara Sharan Nagalur Subraveti $\mathbb{D}^{1,2}$ Victor L. Knoop $\mathbb{D}^{1},{ }^{1}$ and Bart van Arem $\mathbb{D}^{1}$ \\ ${ }^{1}$ Department of Transport and Planning, Delft University of Technology, Delft 2628 CN, Netherlands \\ ${ }^{2}$ Peek Traffic B.V., Basicweg 16, 3821, Amersfoort, Netherlands
}

Correspondence should be addressed to Hari Hara Sharan Nagalur Subraveti; hari.nagalursubraveti@dynniq.com

Received 1 July 2021; Revised 13 December 2021; Accepted 14 December 2021; Published 27 December 2021

Academic Editor: Long Truong

Copyright (@) 2021 Hari Hara Sharan Nagalur Subraveti et al. This is an open access article distributed under the Creative Commons Attribution License, which permits unrestricted use, distribution, and reproduction in any medium, provided the original work is properly cited.

\begin{abstract}
Control measures at merging locations aimed at either the mainline traffic or on-ramp traffic do not lead to a fairness in the distribution of total delay across the two streams. This paper presents a control strategy of combining a lane change control with a ramp metering system at motorway merges. The control strategy presents the opportunity to control the delays incurred at the two traffic streams of the merge. An optimization problem is formulated for a multilane motorway with an on-ramp with the aim to minimize the total travel time of the system. The proposed strategy is tested using an incentive-based lane-specific traffic flow model. Results revealed a $17 \%$ reduction in the total travel time due to the proposed strategy. Moreover, it was shown that the distribution of delays over the mainline and on-ramp could be controlled via the proposed strategy. The performance of the combined control was also compared to the individual control measures. It was observed that the individual control measures (lane change only and ramp metering only) lead to high delays on either the mainline or on-ramp compared to the combined control, where the balance between the delay for the drivers on the mainline and on-ramp could be regulated. The combined lane change and ramp metering control presents opportunities for the road authorities to manage the total delay distribution across the two traffic streams.
\end{abstract}

\section{Introduction}

Motorway merging sections are recurrent bottlenecks prone to congestion due to the high lane changing (LC) activity near the bottleneck with on-ramp and mainline traffic competing for the same space downstream of the merge. This leads to the onset of congestion and a drop in the queue discharge rate, a phenomenon known as capacity drop $[1,2]$. Early attempts to prevent congestion at merging sections are related to the control of on-ramp flow entering the mainline via ramp metering (RM) to avoid or delay the onset of congestion on the mainline $[3,4]$. RM is a popular and wellknown traffic control measure employed at on-ramp sections to deal with congestion problems [5]. RM works by restricting the flow entering the motorway from the onramp. This is usually done by using a traffic light, which controls the ramp flow entering the motorway based on the traffic conditions on the motorway. A variety of RM algorithms have been proposed over the years such as the wellknown feedback based ALINEA [3], feed-forward based ALINEA [6], reinforcement learning based RM [7], RM using microscopic gap detection [8], and RM algorithm based on neural networks [9]. These studies have indicated the benefits of RM. Classical RM algorithms generally work by comparing a measured or target variable such as flow, occupancy, or density of the motorway against a desired/ reference value to avoid the onset of congestion on the motorway. The benefits of RM mainly in terms of reduced travel times have been highlighted in these studies. However, one of the major drawbacks of RM is that, by limiting the onramp flow entering the mainline, long queues can built up on the on-ramp, which can lead to high delay for the on-ramp vehicles. This can also affect the adjacent surface streets due to queue spillback from the on-ramp [10, 11]. This is 
especially true when the on-ramp and mainline demand are high. An overview of various RM algorithms is given by $[5,12]$.

Variable Speed Limit (VSL) is a popular mainstream traffic flow control measure, which is used to regulate the inflow to critical sections and prevent high densities. Multiple studies have used VSL to improve the traffic flow efficiency at merge bottlenecks. VSL controllers such as Model predictive control (MPC) based [13], shockwave theory based [14], feedback based [15], and optimal control based [16] have been tested at merge bottlenecks to improve the traffic flow efficiency. These studies reported certain improvements in travel time and stability. However, the observed improvements in traffic efficiency and travel times are varying and inconsistent $[17,18]$. And in some cases, the designed speed limits are too low $(\sim 20-30 \mathrm{~km} / \mathrm{h})$ to be implemented in practice on the motorway as these can create an additional bottleneck upstream of the VSL $[19,20]$.

Mandatory and suboptimal LCs are among the major reasons for reduced traffic flow efficiency at merging bottlenecks $[10,21]$. Emerging technologies in the form of Vehicle-to-Infrastructure (and Vehicle-to-Vehicle) communication and Advanced Driver Assistance Systems (ADAS) create opportunities to develop smart traffic management strategies, which can alleviate congestion at recurring bottlenecks such as merging sections. Using such emerging technologies, various studies evaluated LC control measures to improve traffic flow efficiency at merging locations. Studies involving LC control are mainly restricted to advisory systems, which use microscopic simulations to analyze the impact of LC advisories to individual vehicles on the motorway. Marinescu et al. [22] proposed a slot-based merging algorithm for automated vehicles, where vehicles are allocated in virtual slots, and the on-ramp vehicles are slotted into any empty slots remaining on the mainline. The algorithm increased the merging probability by utilizing the free slots on the left lanes of the motorway. Park and Smith [23] developed a LC advisory, which encouraged early LCs for the vehicles on the mainline to create more space for merging. Most of the LC advisory studies are usually based on the gap-acceptance approach, where vehicles are advised to change to adjacent lanes when gaps of sufficient size are available and create space to facilitate the merging process. However, the influence of the controlled or advised LCs on traffic flow and any induced LCs was rarely discussed.

Compared to the limited literature on LC advisory systems, there exist several studies that consider the longitudinal control of individual or a platoon of vehicles (either by influencing speed, headway, or acceleration) in order to create gaps near merging sections. Ran et al. [24] and Kato et al. [25] evaluated merging algorithms for fully automated traffic with control over a platoon of vehicles. Davis [26] and Liu et al. [27] investigated the performance of a merging assistant for mixed traffic consisting of manually driven and Cooperative Adaptive Cruise Control (CACC) vehicles. Pueboobpaphan et al. [28] and Nagalur Subraveti et al. [29] developed merging assistants involving speed interventions for the mainline vehicles to create gaps to facilitate merging. Min et al. [30] proposed a centralized approach based on game theory to control the process of onramp merging in a connected environment. However, most of these studies were either limited to single lane motorways or LC behavior, and their impacts were not taken into consideration.

Integrated and coordinated control approaches involving a combination of some of the above-mentioned measures have also been developed. Hegyi et al. [13] used an MPC based controller for optimal coordination of VSL and RM. The framework was evaluated using the second-order METANET model. Carlson et al. [31] integrated VSL with $\mathrm{RM}$ and formulated an optimal control problem to evaluate the performance also using METANET. Iordanidou et al. [32] extended the approach considering multiple on-ramp sections with the aim to balance the delays caused by different actuators. Zhang and Ioannou [18] combined a LC control with VSL near lane closures (merging process somewhat similar to those near on-ramp sections). The VSL controller was based on a feedback linearization technique, while the LC controller provided recommendations to the drivers. However, the LC strategies designed in this study were based on case specific rules, which may not be optimal. Li et al. [33] integrated a local and coordinated RM with VSL controllers to achieve an efficient and equitable motorway system. Tajdari et al. [34] integrated RM with a feedbackbased LC controller and evaluated the performance of the integrated control at merging section assuming the presence of connected and automated vehicles. But the LC flows being regulated were bound to a threshold value instead of the capacity of the target lane. Cho and Laval [35] proposed a RM-VSL combined control strategy to avoid or reduce the extent of capacity drop at merge bottlenecks. However, the study did not assume a minimum speed limit for the VSL or take into account the differences between lanes. Most of these studies also do not discuss the impact of integrated and (or) coordinated control on the distribution of delays across the two traffic streams.

Capacity drop at merging sections is primarily due to the high LC activity-forced and courtesy LCs at these locations. This can be improved by encouraging more discretionary and courtesy LCs further upstream of the on-ramp, so that the conflicts between mainline and ramp traffic are avoided by creating space for the incoming demand. As discussed above, RM alone is not efficient for high traffic demand. And while LC control alone on the mainline can create space for the on-ramp demand, this can cause excessive delays for the mainline traffic due to disturbances and congestion created on the left (inner) lanes due to the LC activity from the right. The unfair allocation of benefits and high delays for on-ramp flow due to RM have been highlighted in multiple studies [36-38]. Restricting to a single control at merging sections leads to an unbalanced distribution of delays across the two traffic streams.

Hence, addressing this issue, the main goal of this study is to combine a LC control with a RM system at merging sections and evaluate the control strategy in terms of the travel time improvements and delay distribution. By combining the LC control with the well-known RM, it is expected that the delays on the mainline and on-ramp can be 
controlled according to the respective demands and prevailing traffic conditions. This paper evaluates the performance of a combined control with the aim to reduce the travel times and improve the traffic flow efficiency at a motorway merging section. The proposed control strategy to manage delays at merging areas is applied on a simple yet realistic test case to show the validity of the concept proposed. Lateral flows upstream of the merge on the mainline are controlled to create more space for the on-ramp demand, and this is coordinated with a RM system to control the onramp demand entering the motorway. The combined control measure is compared to the individual control measures to assess the differences in performance. An optimizationbased framework is used to determine the LC rates upstream of the merging area for the LC control. The various control strategies are evaluated via simulation experiments using an incentive-based first order lane-specific traffic flow model. The traffic stream is considered macroscopically in this study. It should be highlighted that the main contribution of this paper is not the control approach itself but rather the insights obtained via the application of this control approach. Insights gained from this study aim at providing a foundation for the development of traffic management strategies via in-vehicle information systems (IVIS) and roadside units (RSU) for motorway merges.

The remainder of the paper is organized as follows: Section 2 gives a brief description of the lane-specific traffic flow model used for simulation experiments to evaluate the various cases along with a description of the RM algorithm used in this study. In Section 3, description of the optimization problem and the framework for LC control is provided. Section 4 presents the hypothetical network considered along with a description of the no-control scenario. In Section 5, a comparison of the travel times and delays for the different cases is shown, followed by discussions on the observed results, which are reported in Section 6. Finally, the paper is concluded with recommendations for future works in Section 7.

\section{System Modelling}

In this section, a brief description of the traffic flow model used for the simulations and the algorithm used for RM is provided.

2.1. Lane-Specific Traffic Flow Model. An incentive-based lane-specific traffic flow model is used for the simulation experiments to evaluate the performance of the proposed control measure. For a detailed description of the model, we refer to Nagalur Subraveti et al. [39]. The remainder of this section is based on Nagalur Subraveti et al. [39] and provides a summary of the lane-specific model presented in that paper.

The starting point of the model is the well-known cell transmission (CTM) model proposed by Daganzo [40], which is extended to consider lane dynamics. The computation of lateral flows is based on an incentive framework, where LC is described as a function of various incentives such as keep-right, maintaining desired route and courtesy. The model also takes into consideration downstream conditions in the computation of lateral flows. The lateral and longitudinal flows are computed assuming a triangular fundamental diagram (FD). The lanes on the motorway are partitioned into cell segments of length $\Delta x$ and time is discretized into steps of duration $\Delta t$. The density update equation of a multilane motorway divided into lane-wise segments, with the segments indexed as $i=1,2,3, \ldots n$ and the lanes as $l=1,2, \ldots . m$ in discrete terms is

$$
k_{i l}(t+1)=k_{i l}(t)+\frac{\triangle t}{\triangle x}\left[q_{i-1, l}(t)-q_{i l}(t)+l q_{i, l-1 \longrightarrow l}(t)+l q_{i, l+1 \longrightarrow l}(t)-l q_{i, l \longrightarrow l-1}(t)-l q_{i, l \longrightarrow l+1}(t)+r q_{i l}(t)\right] .
$$

In (1), $q$ and $k$ denote the flow and density of the cell segments, respectively. $l q$ is the lateral flow between the cell segments, $r q$ is the flow entering from an on-ramp, and $t$ represents the simulation horizon with $t=1,2,3, \ldots ., \mathrm{T}$. The total simulation time is given by $t_{\text {sim }}=T \Delta t$.

The probability of LC is considered as a function of multiple incentives $(I)$, which are difference in density among lanes $\left(I_{\triangle k}\right)$, desire to maintain route $\left(I_{k r}\right.$, keep-right bias $\left(I_{R}\right.$, and cooperation towards merging vehicles ( $I_{\text {coop }}$, which have been observed in various studies.

$$
I=I_{\triangle k}+I_{k r}+I_{R}+I_{\text {coop }}
$$

The probability of flow to change lanes from $l$ to $l^{\prime}$ is given by

$$
P_{i, l \rightarrow l^{\prime}}=\max \left[0, \frac{I K_{l}-K_{l^{\prime}}}{K_{l}+K_{l^{\prime}}}\right]
$$

where $K_{l}$ is the weighted density of a cell segment $i$ on lane $l$. A weighted density term is considered to take into account the effect of downstream conditions on LC flows. The lateral demand of a cell is a function of $P_{i, l \rightarrow l^{\prime}}$ and the total demand of the cell. The lateral flow $l q_{i, l} \rightarrow l^{\prime}$ in (1) is hence the minimum of the lateral demand of the origin cell and supply of the target cell.

In the model, the flow entering from the on-ramp is prioritized over the longitudinal and lateral flows. This means that the mainstream congestion does not spill back onto the on-ramps, and the on-ramp demand upon entering the acceleration lane can successfully enter the mainline. Empirical studies such as Bar-Gera and Ahn [41] have indicated that the merging behavior is dictated by a fixed ratio denoted as the merge-ratio, which can be equal to the laneratio of the two streams. However, it has been pointed out that there are many other factors influencing this behavior 
(such as lane ratios, road geometry, and curvature). Hence, for simplicity, we make this assumption to preserve the focus of this paper, which are the insights obtained from the implementation of this control strategy.

The first-order traffic flow model is extended to incorporate capacity drop. This is done by employing the approach proposed by Han et al. [42], wherein the supply of a cell segment is decreased with increasing density of the upstream cell segment. This implies that as the cell segment $i$ becomes overcritical, the maximum possible flow in the supply function of the downstream cell segment $i+1$ is linearly decreased as a function of density of cell segment $i$ which results in a flow lower than the capacity of the cell segment.

The traffic flow model has been tested against real-world data for different motorway sections in Netherlands, and it was shown that the model was able to replicate the important lane-specific dynamics (in terms of the lane flow distribution and merging activity) at these sections with a mean error of $\sim 2-3 \mathrm{veh} / \mathrm{km} /$ lane in terms of estimating the lane-specific densities. The model was also compared to a linear regression model, and it was observed that the incentive-based model resulted in lower errors in terms of estimating the lane densities and overall better accuracy. Hence, this model is chosen as a benchmark to represent the no-control scenario against which any performance gains resulting from control are compared.

2.2. Ramp Metering (RM) System. The aim of a RM system is to restrict the on-ramp demand entering the motorway or spread it over time to avoid congestion on the motorway. The benefits of using RM at motorway merge sections to improve traffic flow efficiency and stability have been observed in multiple studies [5, 10]. ALINEA proposed by Papageorgiou et al. [3] is one of the most popular local feedback control RM strategies used. The algorithm uses the occupancy downstream of a $\operatorname{ramp}(\%) o_{\text {out }}(t-1)$ as input for the control strategy. The algorithm works by controlling the ramp inflow to the motorway, such that the occupancy on the mainline is maintained close to a desired value $\widehat{o}$ described by the feedback equation:

$$
r(t)=r(t-1)+K_{R}\left[\widehat{o}-o_{\text {out }}(t-1)\right],
$$

where $r(t)$ denotes the ramp flow allowed to enter the mainline in a time period $(t)$ and $K_{R}$ is a regulator parameter. Several variations of ALINEA have been proposed, where the occupancy term, which is the target/measured, is replaced by other variables such as upstream occupancy, speed, and flow $[4,43]$. In this study, D-ALINEA, which uses density as the measured/targeted variable, is used for the RM system [44]. This is desirable, as the density is the state variable of the traffic flow model described in Section 2. In D-ALINEA, $r(t)$ is given by the feedback equation:

$$
r(t)=r(t-1)+K_{R}[\widehat{k}-k(t-1)],
$$

where $\widehat{k}$ is the desired density, which is related to the critical density as $\widehat{k}=\xi k_{c r}$ with $\xi \leq 1$. $\xi$ can be interpreted as the fraction of the capacity of the road that can be filled [45]. In D-ALINEA, the regulator parameter has a unit of $\mathrm{km} / \mathrm{h}$. The algorithm in this case attempts to prevent the density at the bottleneck from exceeding the critical density. In this study, we assume $\xi=1$. The density measurement used in (5) is the density on the mainline downstream of the acceleration lane. Smaragdis and Papageorgiou [4] mention that it is preferable to control the traffic based on the conditions downstream of the ramp for the best results. Hence, this site was chosen as the area for density measurement. The minimum allowable flow due to RM in this study is set to 300 $\mathrm{veh} / \mathrm{h}$ to prevent long queues upstream of the acceleration lane and avoid ramp closure.

\section{Approach}

This section presents the optimization problem including description of the objective function followed by the framework for the LC control.

3.1. Objective Function. The optimization problem finds LC flows, which lead to the least overall Total Travel Time (TTT) of the system. Since the traffic flow model is based on the demand and supply concept, the flow entering a cell segment will be lower than the demand if the origin cell segments of the network are congested. This will result in a flow, which is less than the demand entering the network, which can hence cause the TTT within the section to be low. This however results in high delays at the entrance of the section. Hence, delays at the entrance of the mainline and on-ramp are also added to the computation of TTT of the system. The objective function aims to minimize TTT, which is equivalent to maximizing throughput and is specified as

$$
J=\int_{0}^{t} N_{i} \Delta t+\int_{0}^{t} N_{m} \Delta t+\int_{0}^{t} N_{r} \triangle t,
$$

where $N=$ number of vehicles in the section $i, N_{m}$ and $N_{r}$ $=$ vehicles queuing at the origin of the mainline and onramp, respectively, due to congestion in the origin cell segments of the network. $\Delta t=$ simulation time step and $t$ $=$ represents the simulation horizon with $t=1,2,3, \ldots, \mathrm{T}$.

The MATLAB implementation of the Sequential Quadratic Programming (SQP) algorithm (fmincon) is used to solve the optimization problem. The LC flows observed in the no-control scenario resulting from traffic flow model described in the previous section are chosen as the initial point for optimization.

3.2. LC Control Framework. We employ the LC control framework proposed in Nagalur Subraveti et al. [46] in this study. For a detailed description of the framework, the authors refer to the original work. The optimization algorithm attempts to find ideal LC flows to minimize the TTT described by (6). The LC flows can be controlled in both directions (i.e., from left to right and right to left). In the case of on-ramps, controlling the LCs from left to right are not of high importance, because vehicles in general would be 
apprehensive to change lanes to the right near merging sections. Hence, for simplicity, only lateral flows towards the left are controlled, and LCs from left to right upstream of the on-ramp are considered to be negligible (zero in this case).

The decision variable chosen for the optimization problem is the probability of flow that can change lanes $\left(P_{l \longrightarrow l^{\prime}}\right)$ described in (3). Selecting $P_{l \rightarrow l^{\prime}}$ as the decision variable is advantageous because of its direct relation to the computation of the LC flows. The constraints for this decision variable can also be easily set since the probability can only range from zero to one. The LC flows are controlled on the mainline in a section upstream of the on-ramp.

\section{Benchmark Problem}

The benchmark network chosen for the simulation experiments is depicted in Figure 1. The network consists of a three-lane mainline motorway with a single lane on-ramp merging into the mainline after $4.5 \mathrm{~km}$. The network is divided into 3 segments. Segment A-B is the mainline section upstream of the on-ramp $4.5 \mathrm{~km}$ long. Segment B-C consists of 3 mainline lanes and an acceleration lane, which is $300 \mathrm{~m}$ long (similar to the length of acceleration lanes commonly observed on Dutch motorways). The section downstream of the acceleration lane is labelled as segment $\mathrm{C}-\mathrm{D}$, which is $1.2 \mathrm{~km}$ in length. The point where the on-ramp intersects with the mainline motorway is labelled as merging point. The RM installation is assumed to be present at this location. The shaded region in the figure on the mainline represents the LC control zone, where the LCs of the mainline traffic are controlled.

The demand profile for the mainline and on-ramp chosen for this study is shown in Figure 2. Both demand profiles follow a similar trend. The simulation is run for 80 minutes with the inflow entering the network being stopped after $1 \mathrm{hr}$. This ensures that the entire flow exits the section, so that the final state of the network is similar for the different test cases enabling a fair comparison of the TTT and delays. A constant demand is maintained for ten minutes followed by a gradual rise for the next 10 minutes. This is followed by constant demand at a high level for the next twenty minutes. Due to the increased inflow, congestion sets in upstream of the merging point on the mainline and onramp. The demand drops from this peak and gradually decreases in the next ten minutes before levelling off and remaining constant for the final ten minutes after which the inflow to network is stopped. During the period of high demand, the lanes are at near-critical conditions.

The network parameters used in the traffic flow model are free flow speed $(u)=108 \mathrm{~km} / \mathrm{h}$, wave speed $(w)$ $=20 \mathrm{~km} / \mathrm{h}$ and jam density $\left(k_{\text {jam }}\right)=128 \mathrm{veh} / \mathrm{km}$. The network parameters are assumed to be the same for all the lanes.

The simulation time step for the model is chosen to be $10 \mathrm{~s}$. According to the assumed simulation time step and the free-flow speed, the minimum cell segment length, which follows the CFL condition [47], is $300 \mathrm{~m}$. This is chosen as the length of the cell segments. A total of 45 cell segments are present upstream of the merging point. The acceleration lane consists of one cell segment of length $300 \mathrm{~m}$. The remaining
15 cell segments are downstream of the end of acceleration lane.

Different control strategies are compared to the nocontrol case to analyze the performance of the system in the presence of the implemented control measures. These different cases include (1) LC control, (2) RM, and (3) combined LC and RM control. In the control cases, which include lateral control, it is assumed that LCs can happen from right to left, while zero LC activity is assumed from left to right. No such constraints are assumed on the direction of LC flows in the no-control scenario. The lateral flows are controlled in a section of length $600 \mathrm{~m}$ upstream of the merging point. No LCs other than the controlled ones are assumed in the optimization problem to make sure that the LC flows resulting from the traffic flow model do not interact with those determined by the optimization process. Note that this can be implemented in practice with a ban on LCs unless instructed otherwise. The control time step for the LC flows is chosen to be 1 minute. Flows entering from the onramp are also controlled via the RM every minute. The density measurement used in equation (5) is the density on the mainline near $\mathrm{C}$ (end of the acceleration lane) in Figure 1. There are a total of six cell segments (two on each lane) within the LC control section.

\section{Results}

In this section, we discuss the results observed in the different control strategies described in the previous section. A comparison between the TTT and delays on the two traffic streams for the different cases and the unsatisfied demand upstream of the on-ramp is shown in this section. A detailed discussion on the observed traffic dynamics along with reasons for the differences in the observed performance is provided in Section 6.

5.1. Comparison of TTT and Delays. In order to get an understanding of the performance of the various control measures evaluated, the TTT of the system as well as the TTT only on the mainline for the different cases is initially looked into. Figure 3 shows the comparison of the no-control scenario against the various control cases in terms of the TTT of the system.

It can be seen from the figure that all the control cases lead to a reduction in the TTT of the system compared to the no-control scenario. The maximum benefits are obtained in the combined LC + RM control case followed by the LC only case and RM only case, respectively. The TTT of the system is reduced by a maximum of $17 \%$ in the $\mathrm{LC}+\mathrm{RM}$ case and a minimum of $4 \%$ in RM only case. While RM leads to significant reduction in the TTT on the mainline, this also leads to high delays for the on-ramp traffic, which can be seen from higher overall TTT of the system compared to the other two control cases.

Now, we take a closer look at the distribution of the total delays incurred upstream of the merge location to get a better understanding of the location of the delays. Figure 4 shows a comparison of the delays at the two traffic streams 


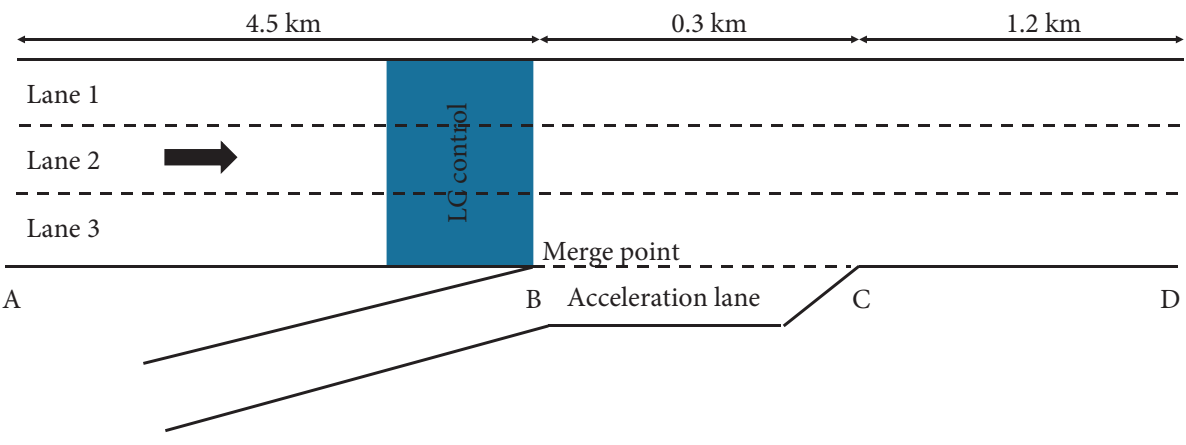

FIgURE 1: Benchmark network.

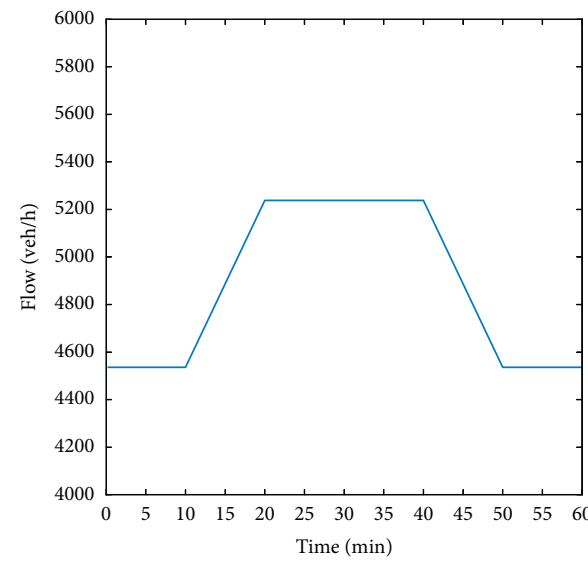

(a)

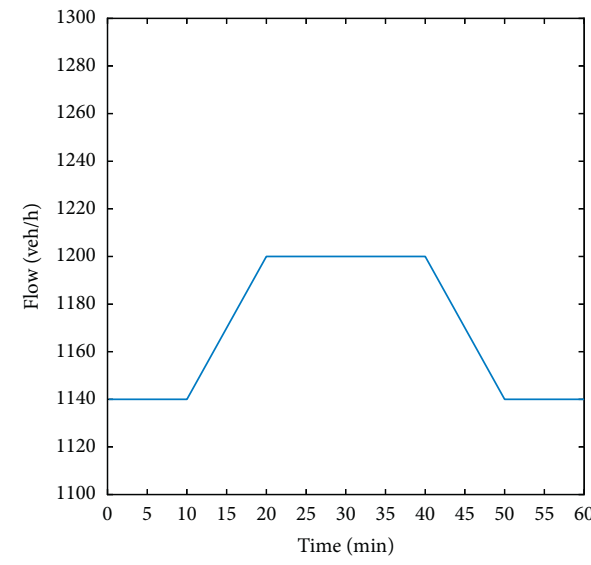

(b)

Figure 2: Demand profiles-mainline demand (a) and on-ramp demand (b).

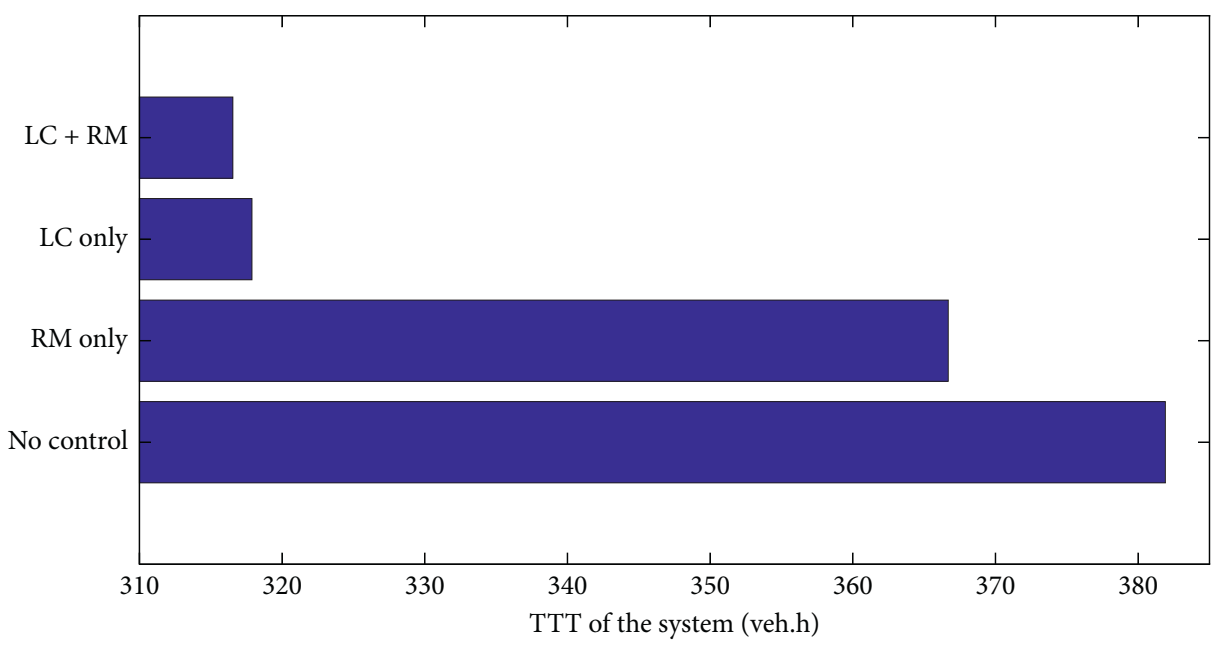

Figure 3: Comparison of the TTT of the system.

upstream of the merging point. In the no-control case, the mainline traffic experiences high delay as the on-ramp demand merges into the mainline. An opposite trend is observed in the RM case where the on-ramp delay is high with slight delay on the mainline. This is to be expected as the onramp demand is restricted from entering the mainline by the
RM system based on the conditions on the mainline. The LC control leads to the least total delay among all the cases although the mainline suffers from a comparatively high delay compared to the RM only case. Compared to the LC only control case, the combined control experiences a slightly higher overall delay. However, the distribution of 


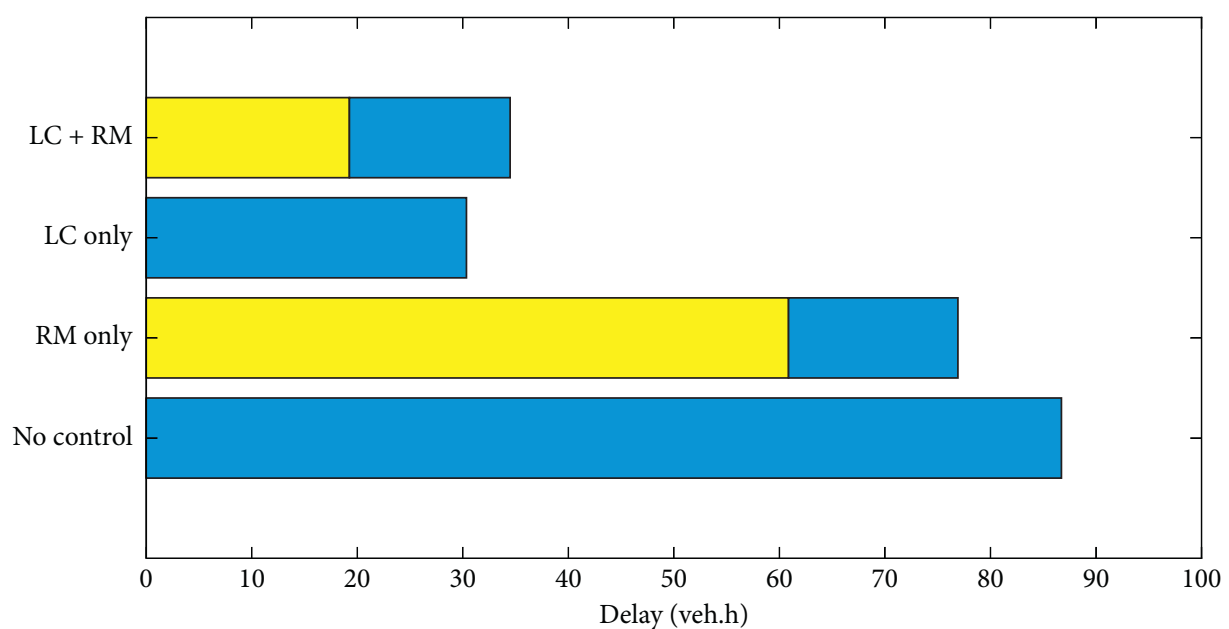

On-ramp delay

Mainline delay

FIGURE 4: Delays upstream of the merging point on the two streams.

delays across the mainline and on-ramp is more even with both experiencing almost similar delays.

The flow exiting the bottleneck gives an insight into the reason behind the variation in the TTT for the different cases. The flows exiting the merge bottleneck (i.e., the location where the acceleration lane ends) are shown in Figure 5. The bottleneck throughput is shown from the $11^{\text {th }}$ minute as the network is in free flow in the first ten minutes. In all the control cases, the exit flow during the period of peak demand is higher than the exit flow in the no-control case. In the traffic flow model, capacity drop is incorporated by modifying the supply function of the cell segment, such that a cell segment is set to receive lower flows when the density of the upstream cell segment becomes overcritical. Since all the control cases reduce conflicts between the mainline and on-ramp traffic by either restricting on-ramp demand via $\mathrm{RM}$ or creating space on the mainline by diverting the mainline flow towards the left lanes via LC control or by using a combination of both, lower densities are observed at the bottleneck area leading to higher exit flows. The combined control leads to higher exit flows for a longer duration as compared to the LC only case. This is because the density on the left lanes starts increasing due to LCs from the right. However, in the combined control case, the number of LCs is lower as part of the on-ramp demand is restricted by the RM system leading to the reduced need for creation of space for the metered on-ramp demand. Fluctuations in the exit flow can be observed in the LC only case along with the combined control case. This is due to the optimizer balancing the need to accommodate ramp demand by directing high LC flows towards left with the need to avoid congestion in the left lanes due to the LC activity. These fluctuations can be avoided by introducing a term that penalizes abrupt variations in the LC flows in the objective function.

5.2. Speed Contour Plots. Figure 6 shows the speed contour plots for the various control cases. We chose to plot pace (i.e., 1/speed) to better illustrate the differences between the low speeds (so technically speaking, they are pace contour plots). However, the corresponding speeds are represented on the color bar for easy interpretation. Note that the differences in pace also better illustrate the differences in travel time than differences in speed. The duration and area of severe congestion (highlighted by the region in red representing lower speeds) are reduced in all the control cases when compared to no-control scenario. In the no-control scenario, as the demand increases on the mainline and onramp, the density on the mainline also increases causing the onset of congestion starting from lane 3 , which then propagates to the left lanes. When the demand on the mainline and on-ramp gradually decreases, the congestion is dissolved, and the network returns to a steady state.

Reduction is speeds observed in the RM only case near the merge point as the traffic from the on-ramp merges into the mainline. However, the congestion does not propagate far upstream and is much less severe as the RM restricts the demand entering lane 3 . In all the cases, congestion begins around the $20^{\text {th }}$ minute, which corresponds to the increase in demand on the mainline and on-ramp. Although there are some courtesy LCs in the no-control and RM cases from lane 3 to the right lanes (lanes 1 and 2) to accommodate the incoming flow, it is not enough due to the considerably high demand.

In the LC control case, the reduction in severe congestion can be attributed to the reduction in the number of conflicts between the mainline and on-ramp vehicles. This is because of the increased number of LCs upstream of the merging point to create space for the incoming on-ramp demand. The net total number of LCs (i.e., difference in the number of LCs in each direction) from lane 3 to 2 is 707 , and from lane 2 to 1 , it is 439 due to the LC control. Comparing this to the no-control case, the net total number of LCs in from lane 3 to 2 is 396 and 191 from lane 2 to 1 . So, the number of LCs from right to left roughly doubles in the LC control case. In the LC only case, more flow is directed 


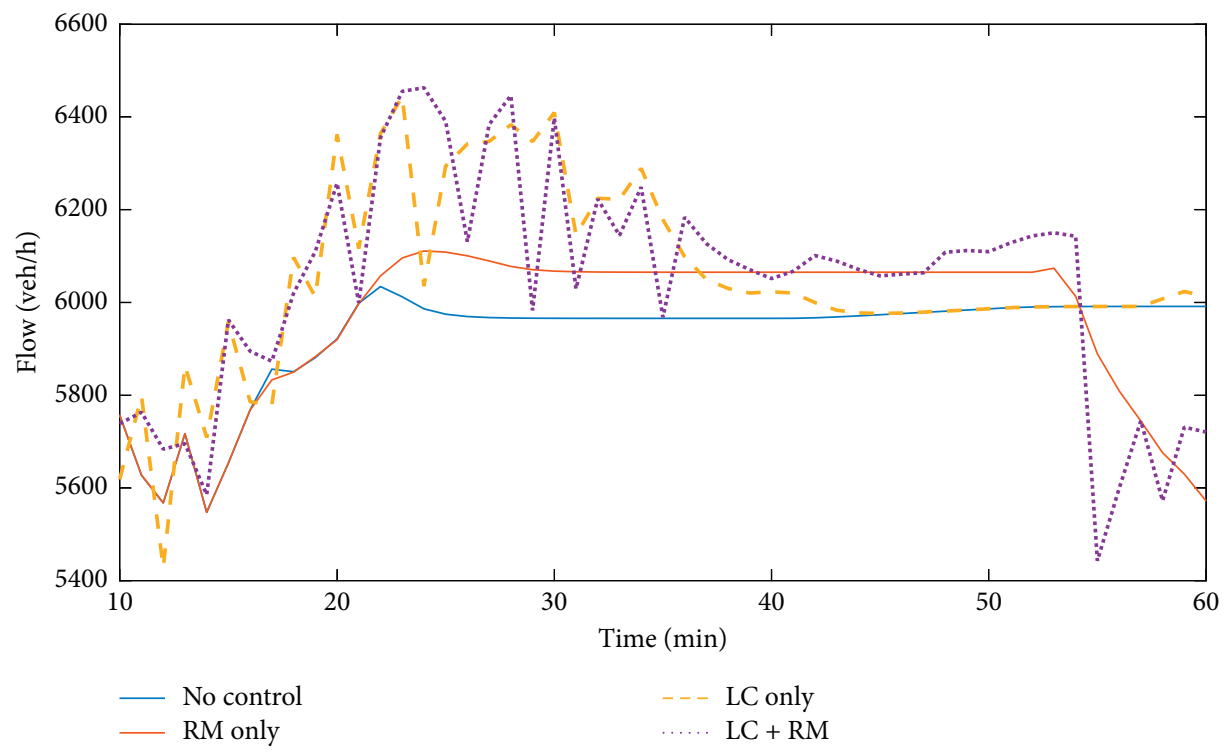

Figure 5: Flow exiting the bottleneck.
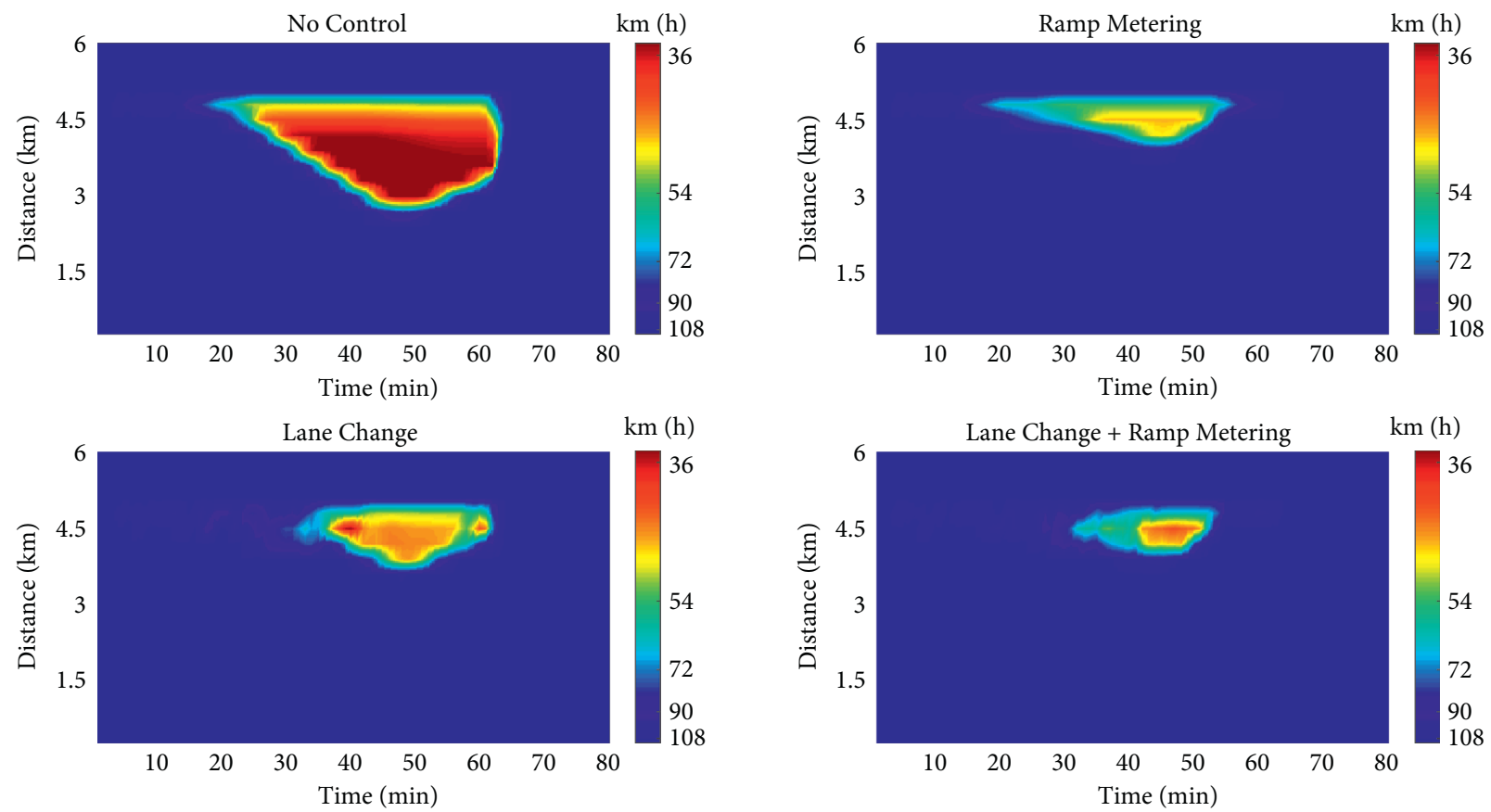

Figure 6: Pace (h/km) contour plots for the mainline. (a) No-control. (b) Ramp metering. (c) Lane change. (d) Lane change+Ramp metering

towards the left lanes to facilitate the merging process by creating gaps. However, this high LC activity causes disruptions and results in mild congestion on the left lanes of the mainline. This causes delay to the traffic on the mainline. This is highlighted in the pace contour plots, where lower speeds are observed compared to the RM only case especially after the $35^{\text {th }}$ minute. However, due to the space created on lane 3 , vehicles coming from the on-ramp can merge more easily into the mainline leading to less delay. A longer LC control section can lead to the LC activity to be more spread out over space probably resulting in lower delays on the mainline. However, in reality, LCs too far upstream of the merging point to create space for the on-ramp flow can be unrealistic and might lead to flow moving back to the right lanes due to increased density in the left lanes.

In the combined control setup, the RM controls the inflow to the mainline via the RM algorithm given by (5), while the space for this metered flow is created via the LC control, where the mainline flow on the right lanes is directed to the left lanes. For the chosen demand profile, as the 


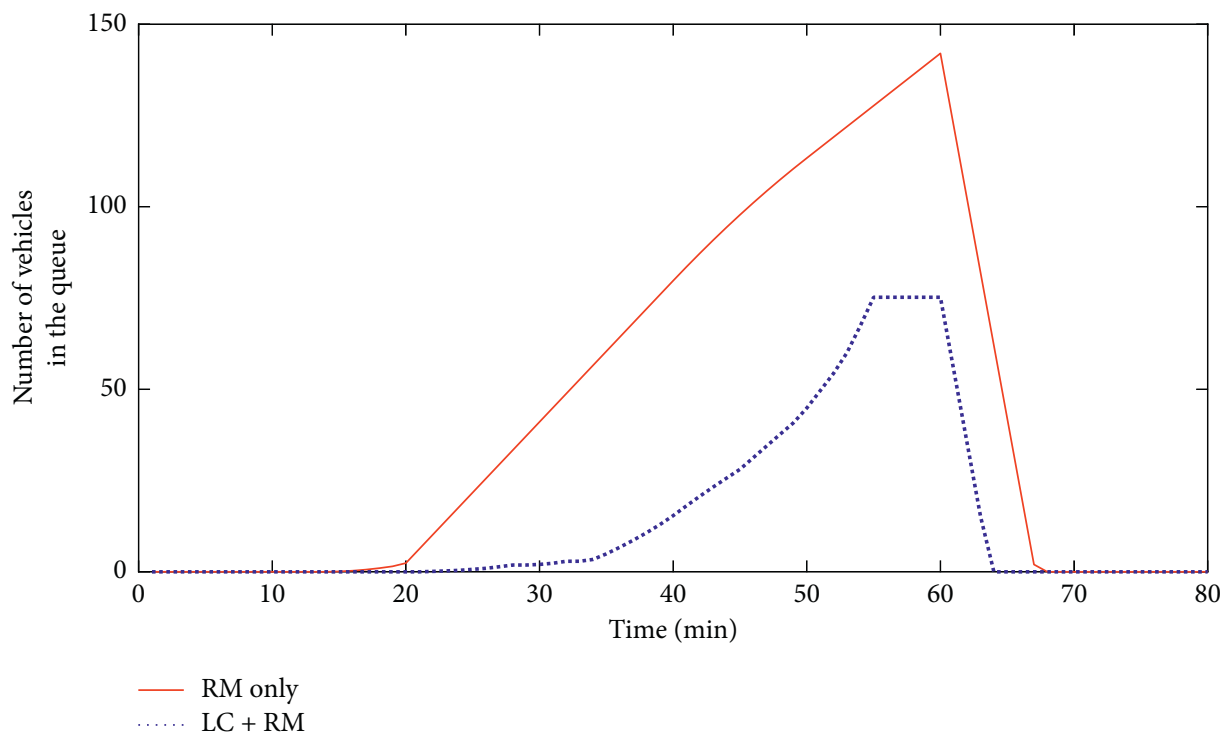

Figure 7: Queue length at the on-ramp.

ramp and mainline demands increase simultaneously at the same rate, the LC rates obtained via the optimization framework and the RM settings led to approximately similar delays upstream of the mainline and on-ramp. There are some differences in the severity of congestion especially near the merging point between the combined and LC cases. In the combined control case, the area of congestion is further reduced especially at the onset of congestion due to the flow that is controlled by the RM, which reduces the number of conflicts between the on-ramp and mainline flow.

5.3. Queue Length at the on-Ramp. Figure 7 shows the queue upstream of the merging point at the on-ramp caused by vehicles that cannot enter the acceleration lane due to the RM system. During the peak demand, the queue length is high and increasing over the entire duration in the RM only case. The duration as well as the peak of the queue is much lower in the combined control case than in the RM only case. This leads to the lower on-ramp delays in the combined control case. In the combined control scheme, the queue length is never too high, which can be beneficial in controlling the queues on the on-ramps. This exhibits the advantages presented by the combined control case as compared to the RM only case. A queue control mechanism wherein the metering rate depends upon the maximum storage space available at the on-ramp is not considered in this study.

\section{Discussions}

In this section, discussions on the results for the various control measures and the reasons for the differences in the observed performance are provided.

The RM case does not yield any significant improvements in terms of the overall TTT when compared to the LC only and combined control case. The reduction in the TTT for the RM case is around 4\% compared to the nearly $17 \%$ reduction in TTT for the other cases. There are two reasons for the lower benefits of the RM case.

Firstly, vehicles from the acceleration lane merge into the mainline initially in lane 3 (also known as the shoulder lane/ right-most lane) and based on the prevailing traffic conditions, and they either remain on this lane or change to the left lanes. Therefore, even if there is enough overall space in the mainline, the available space that is of most importance is the space in lane 3 . The RM system considers the overall available space via the density of the section measured on the mainline at the end of the acceleration lane. While the feedback controller leads to a reduction in flow entering the mainline during overcritical conditions, this flow might still be higher than the space available in lane 3. The major LC activity occurs from the acceleration lane to lane 3 . This leads to the onset of congestion in lane 3 , which slowly propagates to the other lanes as vehicles move to the left lanes due to the sharp increase in density on lane 3 . In addition, the performance of a RM system is generally evaluated using road level traffic flow models. However, in a lane-specific model, each lane is treated as a separate entity. If the RM system is changed to consider the density of lane 3 only, the flow due to RM is greatly reduced, which causes large queues upstream of the acceleration lane and consequently, lower improvements in TTT. Hence, the availability of space on the right-most lane should be given higher importance, and the prevailing lane flow distribution should be taken into consideration while designing the RM algorithms.

Secondly, it is assumed that RM rates are controlled every minute. The measurement of the target variable, which is the density of the mainline section near the bottleneck, is performed every minute (similar to the aggregation period of roadside loop detectors). Hence, it is possible that, at a particular time interval, the mainline is undercritical. However, in the next interval, due to higher demand (either on the mainline or ramp or in some cases both), the section 
can get overcritical. Especially, at near-critical conditions, even slight increase in demand can quickly lead to the onset of congestion. In the case study, the section changes from near critical to overcritical between the 13th and 14th minutes. Combined with low metering rates, high on-ramp demand, and the control period of the metering rate, the RM is not able lead to sufficiently high exit flows and consequently lower TTT.

The improvements in the TTT in the LC control case were due to the higher number of LCs towards the left lanes spread over space in the LC control section leading to more space for the ramp demand and reduced conflicts between the two traffic streams. The TTT for the combined control was even lower than the LC only case. The total delay in this case was also observed to be distributed evenly on the mainline and on-ramp.

However, the delays across the mainline and on-ramp can also be controlled in this coordinated setup by changing the parameters of the RM and LC control, which can change the distribution of delays. For example, higher rates of RM can result in the flow from the on-ramp to be reduced leading to a comparatively higher delay and queue length at the entrance of the ramp. This leads to a decrease in the number of LCs on the mainline resulting in lower delay on the mainline.

Figure 8 shows the variations of the delays on the mainline and on-ramp for different control parameter values. Delays upstream of the on-ramp are represented on the horizontal axis, and delays on the mainline upstream of the merging point are represented on the vertical axis. The LC rates obtained from the optimization framework are varied along with the metering rate of the ALINEA algorithm. In the figure, more LC rates towards the left lanes on the mainline imply priority towards the on-ramp flow, and mainline priority is indicated by higher metering rate for the on-ramp demand. The delay isolines represent a range between which the overall delays might fall by regulating the control parameters. The mainline delay is highly reduced when the on-ramp demand is highly metered. This is to be expected as there are no disturbances created on the mainline due to the space created on the mainline by the LC control. The conflicts between the two streams are greatly reduced as well due to the control of the on-ramp demand entering the motorway due to the higher metering rate. This, however, leads to extremely high delays for the on-ramp vehicles. When the on-ramp demand is prioritized, the mainline experiences very high delays due to the high LC rates towards the left lanes on the mainline. The number of conflicts between the mainline and on-ramp traffic is reduced (although not completely eliminated), but the increased interactions between the mainline vehicles causes disturbances on the mainline leading to comparatively higher delays on the mainline.

The findings show that the combined LC and RM control allows the possibility to vary the delays across the two streams while also leading to a reduction in the overall TTT of the system. The combined control allows the possibility to consider the distribution of delays and the fairness between the mainline and on-ramps. Thus, based on the incoming

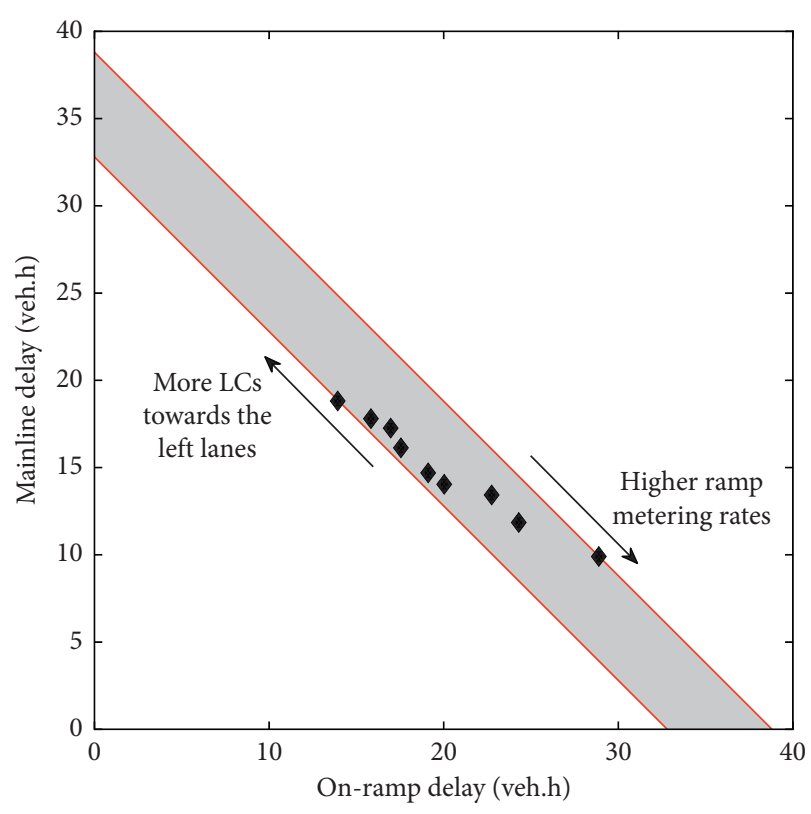

FIgURE 8: Variation of delays across the two streams with control parameters.

demand on the two traffic streams, the coordinated control can be tuned accordingly to avoid heavy congestion on either stream. If the demand on the ramp is high, LC control can be used to direct flow away from the right lanes and create space. And when the mainline demand is high, RM can be used to control the flow entering the mainline.

\section{Conclusions}

The proposed control strategy combines a lane-specific traffic flow model, which has been validated against realworld data, and a traditional and robust RM system to manage the delays at merge sections. The performance of a proposed control strategy is evaluated for near-critical traffic conditions at motorway merges. An optimization problem was formulated to determine the LC rates upstream of the merged area on the mainline of a multilane motorway. This was coordinated with the density-based variation of ALINEA RM system, which used the density downstream of the merge as the measured/targeted variable. The combined control scheme was also compared to the individual control measures to observe the differences in performance. The control measures were evaluated via simulation experiments using an incentive-based lane-specific traffic flow model. It was observed that the combined control case along with the LC control only case resulted in considerable reduction in the total travel time of the network. However, the way the total delay was distributed upstream of the merge varied across the different cases. In the individual control cases, the total delay was disproportionately distributed with high delays on either the mainline or on-ramp. However, the combined control strategy led to similar delays across the two traffic streams. It was also shown that these delays could be regulated by changing the parameters of the combined control setup. The choice in terms of prioritizing between 
mainline and on-ramp control can be made based on their respective demands, which is made possible via the coordinated control.

While the proposed control strategy was successfully validated against a simple yet realistic test case, it is important to investigate the robustness of the coordinated control setup for a variety of demand profiles and traffic conditions. Future works can also include assessing the performance gains using microsimulation tools as well as evaluating the proposed strategies for mixed traffic involving Connected Autonomous Vehicles (CAVs). Furthermore, the study is currently restricted to isolated merges and extensions to multiple on-ramp sections with coordinated LC and RM can also be explored.

\section{Data Availability}

No data were used to support this study.

\section{Conflicts of Interest}

The authors declare no potential conflicts of interest with respect to the research, authorship, and/or publication of this article.

\section{Acknowledgments}

This research was performed in Taking the Fast Lane project, which was funded by 'Applied and Technical Sciences' (TTW)-13771, which is a subdomain of NWO.

\section{References}

[1] M. J. Cassidy and R. L. Bertini, "Some traffic features at freeway bottlenecks," Transportation Research Part B: Methodological, vol. 33, no. 1, pp. 25-42, 1999.

[2] R. L. Bertini and S. Malik, "Observed dynamic traffic features on freeway section with merges and diverges," Transportation Research Record: Journal of the Transportation Research Board, vol. 1867, no. 1, pp. 25-35, 2004.

[3] M. Papageorgiou, H. Hadj-Salem, and J. M. Blosseville, "ALINEA: a local feedback control law for on-ramp metering," Transportation Research Record, vol. 1320, no. 1, pp. 58-67, 1991.

[4] E. Smaragdis and M. Papageorgiou, "Series of new local ramp metering strategies: emmanouil smaragdis and markos Papageorgiou," Transportation Research Record: Journal of the Transportation Research Board, vol. 1856, no. 1, pp. 74-86, 2003.

[5] M. Papageorgiou and A. Kotsialos, "Freeway ramp metering: an overview," IEEE Transactions on Intelligent Transportation Systems, vol. 3, no. 4, pp. 271-281, 2002.

[6] J. R. D. Frejo and B. D. Schutter, "Feed-forward ALINEA: a ramp metering control algorithm for nearby and distant bottlenecks," IEEE Transactions on Intelligent Transportation Systems, vol. 20, no. 7, pp. 2448-2458, 2018.

[7] B. Liu, Y. Tang, Y. Ji, Y. Shen, and Y. Du, "A deep reinforcement learning approach for ramp metering based on traffic video data," Journal of Advanced Transportation, vol. 2021, Article ID 6669028, 13 pages, 2021.

[8] S. R. Klomp, V. L. Knoop, H. Taale, and S. P. Hoogendoorn, "Ramp Metering with Microscopic Gap Detection Algorithm
Design and Empirical Acceleration Verification." Transportation Research Record, vol. 2021, Article ID 03611981211033861, 2021.

[9] Y. Ci, H. Wu, Y. Sun, and L. Wu, "A prediction model with wavelet neural network optimized by the chicken swarm optimization for on-ramps metering of the urban expressway," Journal of Intelligent Transportation Systems, vol. 2021, pp. 1-18, 2021.

[10] M. J. Cassidy and J. Rudjanakanoknad, "Increasing the capacity of an isolated merge by metering its on-ramp," Transportation Research Part B: Methodological, vol. 39, no. 10, pp. 896-913, 2005.

[11] M. K. Shehada and A. Kondyli, "Evaluation of ramp metering impacts on travel time reliability and traffic operations through simulation," Journal of Advanced Transportation, vol. 2019, Article ID 8740158, 12 pages, 2019.

[12] F. Vrbanić, E. Ivanjko, K. Kušić, and D. Čakija, "Variable speed limit and ramp metering for mixed traffic flows: a review and open questions," Applied Sciences, vol. 11, no. 6, p. 2574, 2021.

[13] A. Hegyi, B. De Schutter, and H. Hellendoorn, "Model predictive control for optimal coordination of ramp metering and variable speed limits," Transportation Research Part C: Emerging Technologies, vol. 13, no. 3, pp. 185-209, 2005.

[14] A. Hegyi, S. P. Hoogendoorn, M. Schreuder, H. Stoelhorst, and F. Viti, "SPECIALIST: a dynamic speed limit control algorithm based on shock wave theory," in Proceedings of the 11th International IEEE Conference on Intelligent Transportation Systems, pp. 827-832, IEEE, Beijing, China, October 2008.

[15] R. C. Carlson, I. Papamichail, and M. Papageorgiou, "Comparison of local feedback controllers for the mainstream traffic flow on freeways using variable speed limits," Journal of Intelligent Transportation Systems, vol. 17, no. 4, pp. 268-281, 2013.

[16] R. C. Carlson, I. Papamichail, M. Papageorgiou, and A. Messmer, "Optimal motorway traffic flow control involving variable speed limits and ramp metering," Transportation Science, vol. 44, no. 2, pp. 238-253, 2010.

[17] X.-Y. Lu and S. E. Shladover, "Review of variable speed limits and advisories," Transportation Research Record: Journal of the Transportation Research Board, vol. 2423, no. 1, pp. 15-23, 2014.

[18] Y. Zhang and P. A. Ioannou, "Combined variable speed limit and lane change control for highway traffic," IEEE Transactions on Intelligent Transportation Systems, vol. 18, no. 7, pp. 1812-1823, 2016.

[19] M. Hadiuzzaman, T. Z. Qiu, and X.-Y. Lu, "Variable speed limit control design for relieving congestion caused by active bottlenecks," Journal of Transportation Engineering, vol. 139, no. 4, pp. 358-370, 2013.

[20] C. Zhang, N. R. Sabar, E. Chung, A. Bhaskar, and X. Guo, "Optimisation of lane-changing advisory at the motorway lane drop bottleneck," Transportation Research Part C: Emerging Technologies, vol. 106, pp. 303-316, 2019.

[21] P. Hidas, "Modelling vehicle interactions in microscopic simulation of merging and weaving," Transportation Research Part C: Emerging Technologies, vol. 13, no. 1, pp. 37-62, 2005.

[22] D. Marinescu, J. Čurn, M. Bouroche, and V. Cahill, “On-ramp traffic merging using cooperative intelligent vehicles: a slotbased approach," in Proceedings of the 15th International IEEE Conference on Intelligent Transportation Systems, pp. 900-906, IEEE, Anchorage, AK, USA, September 2012. 
[23] H. Park and B. L. Smith, "Investigating benefits of IntelliDrive in freeway operations: lane changing advisory case study," Journal of Transportation Engineering, vol. 138, no. 9, pp. 1113-1122, 2012.

[24] B. Ran, S. Leight, and B. Chang, "A microscopic simulation model for merging control on a dedicated-lane automated highway system," Transportation Research Part C: Emerging Technologies, vol. 7, no. 6, pp. 369-388, 1999.

[25] S. Kato, S. Tsugawa, K. Tokuda, T. Matsui, and H. Fujii, "Vehicle control algorithms for cooperative driving with automated vehicles and intervehicle communications," IEEE Transactions on Intelligent Transportation Systems, vol. 3, no. 3, pp. 155-161, 2002.

[26] L. C. Davis, "Effect of adaptive cruise control systems on mixed traffic flow near an on-ramp," Physica A: Statistical Mechanics and Its Applications, vol. 379, no. 1, pp. 274-290, 2007.

[27] H. Liu, X. Kan, S. E. Shladover, X.-Y. Lu, and R. E. Ferlis, "Impact of cooperative adaptive cruise control on multilane freeway merge capacity," Journal of Intelligent Transportation Systems, vol. 22, no. 3, pp. 263-275, 2018.

[28] R. Pueboobpaphan, F. Liu, and B. van Arem, "The impacts of a communication based merging assistant on traffic flows of manual and equipped vehicles at an on-ramp using traffic flow simulation," in Proceedings of the 13th International IEEE Conference on Intelligent Transportation Systems, pp. 14681473, IEEE, Funchal, Portugal, September 2010.

[29] H. H. S. Nagalur Subraveti, V. L. Knoop, and B. V. Arem, "Rule based control for merges: assessment and case study," in Proceedings of the 21st International IEEE Conference on Intelligent Transportation Systems, pp. 3006-3013, IEEE, Maui, HI, USA, November 2018.

[30] H. Min, Y. Fang, R. Wang, X. Li, Z. Xu, and X. Zhao, "A novel on-ramp merging strategy for connected and automated vehicles based on game theory," Journal of Advanced Transportation, vol. 2020, Article ID 2529856, 11 pages, 2020.

[31] R. C. Carlson, I. Papamichail, and M. Papageorgiou, "Integrated feedback ramp metering and mainstream traffic flow control on motorways using variable speed limits," Transportation Research Part C: Emerging Technologies, vol. 46, pp. 209-221, 2014.

[32] G.-R. Iordanidou, I. Papamichail, C. Roncoli, and M. Papageorgiou, "Feedback-based integrated motorway traffic flow control with delay balancing," IEEE Transactions on Intelligent Transportation Systems, vol. 18, no. 9, pp. 2319-2329, 2017.

[33] D. Li, P. Ranjitkar, and A. Ceder, "Integrated approach combining ramp metering and variable speed limits to improve motorway performance," Transportation Research Record: Journal of the Transportation Research Board, vol. 2470, no. 1, pp. 86-94, 2014.

[34] F. Tajdari, C. Roncoli, and M. Papageorgiou, "Feedback-based ramp metering and lane-changing control with connected and automated vehicles," IEEE Transactions on Intelligent Transportation Systems, vol. 99, 2020.

[35] H. W. Cho and J. A. Laval, "Combined ramp-metering and variable speed limit system for capacity drop control at merge bottlenecks," Journal of Transportation Engineering, Part A: Systems, vol. 146, no. 6, Article ID 04020033, 2020.

[36] A. Kotsialos and M. Papageorgiou, "Efficiency versus fairness in network-wide ramp metering," in Proceedings of the 2001 IEEE Intelligent Transportation Systems. Proceedings (Cat. No.01TH8585), pp. 1189-1194, IEEE, Oakland, CA, USA, August 2001.
[37] Y. Yin, H. Liu, and H. Benouar, "A note on equity of ramp metering," in Proceedings of the 7th International IEEE Conference on Intelligent Transportation Systems, pp. 497-502, IEEE, Washington, WA, USA, October 2004.

[38] N. Amini, L. Gardner, and S. T. Waller, "New horizontal equity measure for ramp meters," Transportation Research Record: Journal of the Transportation Research Board, vol. 2568, no. 1, pp. 90-102, 2016.

[39] H. H. S. Nagalur Subraveti, V. L. Knoop, and B. V. Arem, "First order multi-lane traffic flow model - an incentive based macroscopic model to represent lane change dynamics," Transportation Business: Transport Dynamics, vol. 7, no. 1, pp. 1758-1779, 2019.

[40] C. F. Daganzo, "The cell transmission model: a dynamic representation of highway traffic consistent with the hydrodynamic theory," Transportation Research Part B: Methodological, vol. 28, no. 4, pp. 269-287, 1994.

[41] H. Bar-Gera and S. Ahn, "Empirical macroscopic evaluation of freeway merge-ratios," Transportation Research Part C: Emerging Technologies, vol. 18, no. 4, pp. 457-470, 2010.

[42] Y. Han, Y. Yuan, A. Hegyi, and S. P. Hoogendoorn, "New extended discrete first-order model to reproduce propagation of jam waves," Transportation Research Record: Journal of the Transportation Research Board, vol. 2560, no. 1, pp. 108-118, 2016.

[43] R. Chi, Z. Hou, S. Jin, D. Wang, and J. Hao, "A data-driven iterative feedback tuning approach of ALINEA for freeway traffic ramp metering with PARAMICS simulations," IEEE Transactions on Industrial Informatics, vol. 9, no. 4, pp. 2310-2317, 2013.

[44] S. Hoogendoorn, R. Landman, J. V. Kooten, and M. Schreuder, "Integrated network management amsterdam: control approach and test results," in Proceedings of the 16th International IEEE Conference on Intelligent Transportation Systems, pp. 474-479, IEEE, The Hague, Netherlands, October 2013.

[45] V. L. Knoop, H. Taale, M. Meulenberg, P. B. V. Erp, and S. P. Hoogendoorn, "Ramp metering with real-time estimation of parameters," in Proceedings of the 21st International Conference on Intelligent Transportation Systems, pp. 36193626, IEEE, Maui, HI, USA, November 2018.

[46] H. H. S. Nagalur Subraveti, V. L. Knoop, and B. V. Arem, "Improving traffic flow efficiency at motorway lane drops by influencing lateral flows," Transportation Research Record Journal of the Transportation Research Board, vol. 2674, Article ID 0361198120948055, 2020.

[47] R. Courant, K. Friedrichs, and H. Lewy, "Uber die partiellen differenzengleichungen der mathematischen physik," Mathematische Annalen, vol. 100, no. 1, pp. 32-74, 1928. 